\title{
HOMOTHETIES AND ISOMETRIES OF METRIC SPACES
}

\section{Mauro Patrão *}

\begin{abstract}
Let $(M, d)$ be a metric space. We prove that when the group of homotheties $H(M, d)$ is a locally compact group, with respect to the compactopen topology, it is a Lie group if, and only if, the group of isometries $I(M, d)$ is a Lie group. Then we prove that when $(M, d)$ is a Heine-Borel metric space, its group of homotheties $H(M, d)$ is also a Heine-Borel metric space and, if $(M, d)$ is a Heine-Borel ultrametric space, its group of isometries is an increasing union of compact subgroups. We also prove that when $(M, d)$ is locally compact and the space $\Sigma(M)$ of the connected components of $M$ is quasi-compact, its group of homotheties $H(M, d)$ is locally compact. As applications we give some generalizations of classical results in Riemannian geometry. Namely, if $(M, d)$ is a Finsler manifold or an Alexandroff space, then its group of homotheties is a Lie group. With some additional hypothesis, this is also true for a Hadamard space.
\end{abstract}

\section{Introduction}

Let $(M, d)$ be a metric space. The group of homotheties $H(M, d)$ is defined by

$H(M, d)=\{f \in C(M): f$ is onto and $d(f(x), f(y))=\lambda(f) d(x, y), \quad \forall x, y \in M\}$,

where $C(M)$ is the set of all continuous mappings of $M$ into itself and $\lambda(f)>0$ is a constant. The group of isometries $I(M, d)=\{f \in H(M, d): \lambda(f)=1\}$ is a closed normal subgroup of $H(M, d)$. It is a classical result of the theory

*I thank the help of my friends L. Seco and L. San Martin and the careful comments of the referee.

Supported by FAPESP: proc. $n^{\circ}$ 02/13839-4

Keywords: homothety group, isometry group, proper action, Lie group, Heine-Borel metric space, ultrametric space.

Mathematics Subject Classification: Primary: 54E40, 54E45; Secondary: 22E99, 54H15. 
of topological groups due to Gleason and Yamabe (cf. [10]) that a topological group $G$ is a Lie group if, and only if, it is locally compact and has no small subgroups. Using this deep result, we prove that when the group of homotheties $H(M, d)$ is a locally compact group, it is a Lie group if, and only if, the group of isometries $I(M, d)$ is a Lie group.

Then we prove that, under some reasonable conditions over $(M, d)$, the group of homotheties $H(M, d)$ is locally compact. Since the work of van Dantzig and van der Waerden [4], it is well known that if $M$ is connected and locally compact then its group of isometries $I(M, d)$ is locally compact with respect to the compact-open topology. Recently, Manoussos-Strantzalos [9] replaced the connectivity of $M$ by the weaker hypothesis that the space $\Sigma(M)$ of the connected components of $M$ is quasi-compact (compact but not necessarily Hausdorff) with respect to quotient topology. Also, Gao-Kechris [13] proved a stronger result about the group of isometries $I(M, d)$ encompassing the above ones.

We prove some extensions and variations of these results for the group of homotheties $H(M, d)$. First we consider Heine-Borel metric spaces, i.e., metric spaces whose the compact subsets are the bounded and closed ones. We prove that for a Heine-Borel metric space $(M, d)$ its group of homotheties $H(M, d)$ is also a Heine-Borel metric space. In particular, it follows that $I(M, d)$ is a HeineBorel metric space. For this result we do not require any further assumption, like e.g. the quasi-compactness of [9]. The set $M=\mathbb{Z}$ of integers with the standard distance $d(x, y)=|x-y|$, is a simple example of a metric space where $\Sigma(M)$ is not quasi-compact but $M$ is a Heine-Borel space, so our results apply to it. As a consequence of our methods we give a new proof of the fact [13] that action of $I(M, d)$ on $M$ is proper if $(M, d)$ is Heine-Borel.

The class of Heine-Borel metric spaces is interesting for two facts. First, because there is a consequence of the Hopf-Rinow theorem for length spaces, (cf. [2]), which claims that $(M, d)$ is Heine-Borel if and only if it is complete and locally compact. Second, because of the following fact: if $(M, d)$ is a separable and locally compact metric space then there is a metric $d^{\prime}$ equivalent to $d$ such 
that $\left(M, d^{\prime}\right)$ is Heine-Borel [8].

We also consider the group of isometries $I(M, d)$ of Heine-Borel ultrametric spaces $(M, d)$, i.e., metric spaces where

$$
d(x, z) \leq \max \{d(x, y), d(y, z)\}
$$

for all $x, y, z \in M$. It is proved that if $(M, d)$ is a Heine-Borel ultrametric space then its group of isometries is an increasing union of compact subgroups. This result is an improvement of a theorem due to Gao-Kechris [13], which states that the group of isometries is the closure of an increasing union of compact subgroups. Also, we prove that if $(M, d)$ is a Heine-Borel ultrametric space and $G$ is a finitely generated subgroup of $I(M, d)$ then $\operatorname{cl}(G)$ is compact . HeineBorel ultrametric spaces are extensively used in Number Theory because of the Ostrowski theorem [3], [12] which states that every nontrivial norm on $\mathbb{Q}$ is equivalent to the standard absolute value or to the Heine-Borel ultrametric $p$-adic norm for some prime $p$.

Next we consider metric spaces $(M, d)$ such that the space $\Sigma(M)$ of the connected components of $M$ is quasi-compact with respect to quotient topology. First we generalize the result of [9] for the group of homotheties: if $M$ is a locally compact metric space and $\Sigma(M)$ is quasi-compact then its group of homotheties $H(M, d)$ is locally compact with respect to the compact-open topology.

In this setting we also look at the quasi-metric spaces, i.e., a space $(M, d)$ where $d$ satisfies all the distance axioms, except perhaps that $d(x, y)$ is not necessarily equal to $d(y, x)$ for all $x, y \in M$, and such that the topology generated by forwards metric balls is equal to the topology generated by backwards metric balls. We prove that if $M$ is a locally compact quasi-metric space and $\Sigma(M)$ is quasi-compact then its group of homotheties $H(M, d)$ is locally compact with respect to the compact-open topology. An interesting example of quasi-metric spaces are the Finsler manifolds, where its Finsler function $F$ is positively homogeneous but not necessary absolutely homogeneous and, for all $x, y \in M$, we define $d(x, y)$ as the greatest lower bound of the length of all smooth curves joining $x$ to $y$. 
As applications we give some generalizations of classical results in Riemannian geometry. If $(M, d)$ is a Finsler manifold or an Alexandroff space, then its group of homotheties is a Lie group. With some additional hypothesis, this is also true for a Hadamard space.

\section{Homotheties and isometries}

Let $(M, d)$ be a metric space. We denote the closed and open metric balls of $(M, d)$ centered in $x \in M$ with radius $r>0$, respectively, by $B[x, r]=\{y \in$ $M: d(x, y) \leq r\}$ and by $B(x, r)=\{y \in M: d(x, y)<r\}$. We also denote the closure of $N \subset M$ by $\operatorname{cl}(N)$. The group of homotheties of $M$ is defined by

$H(M, d)=\{f \in C(M): f$ is onto and $d(f(x), f(y))=\lambda(f) d(x, y), \forall x, y \in M\}$,

where $C(M)$ is the set of all continuous mappings of $M$ into itself and $\lambda(f)>0$ is a constant. The group of isometries of $M$ is defined by $I(M, d)=\{f \in$ $H(M): \lambda(f)=1\}$. We show next that there is a continuous homomorphism from $H(M, d)$ to the multiplicative group $(0, \infty)$ :

Lemma 2.1. The mapping $f \mapsto \lambda(f)$ is a continuous homomorphism, with respect to the compact-open topology, from $H(M, d)$ to the multiplicative group $(0, \infty)$.

Proof. Clearly, for all $f, g \in H(M, d)$, we have $\lambda(f \circ g)=\lambda(f) \lambda(g)$ and $\lambda\left(f^{-1}\right)=\lambda(f)^{-1}$. To see that $\lambda$ is continuous and $H(M, d)$ is closed, with respect to the compact-open topology, let $\left(f_{i}\right)_{i \in I}$ be a net in $H(M, d)$ such that $f_{i} \rightarrow f$ in the compact-open topology, which implies that $f_{i}(x) \rightarrow f(x)$, for all $x \in M$. Let $w, z \in M$ be such that $w \neq z$. Then

$$
\lambda\left(f_{i}\right)=\frac{d\left(f_{i}(w), f_{i}(z)\right)}{d(w, z)} \rightarrow \frac{d(f(w), f(z))}{d(w, z)}=\lambda(f) .
$$

Let $G$ be a subgroup of $H(M, d)$ and denote $[G, G]$ the commutator group, that is, the smallest closed subgroup of $G$ containing all elements of the form 
$[f, g]=f \circ g \circ f^{-1} \circ g^{-1}$. From what was shown above, we obtain the following corollary for the groups $H(M, d)$ and $I(M, d)$ of a metric space $(M, d)$ :

Proposition 2.1. Let $(M, d)$ be a metric space and $G$ a subgroup of $H(M, d)$. Then the following statements holds:

1. $I(M, d)$ is a closed normal subgroup of $H(M, d)$.

2. If $G /[G, G]$ is compact, then $G$ is a subgroup of $I(M, d)$.

3. If $G$ is compact then $G$ is a compact subgroup of $I(M, d)$.

\section{Proof.}

1. Clearly $I(M, d)$ is the kernel of the continuous homomorphism $\lambda$.

2. Since $\lambda$ is a homomorphism and $(0, \infty)$ is an abelian group, it follows that $[G, G] \subset \operatorname{ker}(\lambda)$. Hence, since $\lambda$ is a continuous homomorphism, $\lambda(G)$ is isomorphic to $G / \operatorname{ker}(\lambda)$ and therefore $\lambda(G)$ is a compact subgroup of $(0, \infty)$. Hence $\lambda(G)=\{1\}$ implying that $G \subset I(M, d)$.

3. If $G$ is compact, $G /[G, G]$ is compact.

Since the group of isometries $I(M, d)$ is a closed subgroup of $H(M, d)$ with respect to the compact-open topology most of the results for $H(M, d)$ apply immediately to the group of isometries. A topological group $G$ has no small subgroups if there is a neighborhood $V$ of the identity element $e \in G$ with the following property: if $H \subset V$ is a subgroup of $G$, then $H=\{e\}$. The next result is a consequence of a theorem due Gleason and Yamabe (cf. [15], [10]) which states that $G$ is a Lie Group if, and only if, $G$ is a locally compact group and has no small subgroups.

Theorem 2.1. Suppose that $H(M, d)$ is locally compact. Then $I(M, d)$ is a Lie group if, and only if, $H(M, d)$ is a Lie group. 
Proof. If $H(M, d)$ is a Lie group, then $I(M, d)$ is a Lie group, since $I(M, d)$ is a closed subgroup. Assume that $I(M, d)$ is a Lie group. Then $I(M, d)$ has no small subgroups, i.e., there is a neighborhood $U \subset I(M, d)$ of the identity id in $\mathrm{I}(\mathrm{M}, \mathrm{d})$ such that if $G \subset U$ is a subgroup of $I(M, d)$, then $G=\{\mathrm{id}\}$. Let $V \subset H(M, d)$ be a neighborhood of the identity in $H(M, d)$ such that $U=V \cap I(M, d)$ and $W \subset V$ another neighborhood of the identity in $H(M, d)$ such that $\operatorname{cl}(W)$ is compact. Let $G \subset W$ be a subgroup of $H(M, d)$. Thus $\operatorname{cl}(G)$ is compact and hence $G$ is a subgroup of $I(M, d)$ (cf. Proposition 2.1). Since $G \subset W \cap I(M, d) \subset U, G=\{\mathrm{id}\}$. Therefore $H(M, d)$ is locally compact and has no small subgroups and hence is a Lie group.

In the sections 3 and 5, we prove that, under some reasonable conditions over $(M, d)$, the group of homotheties $H(M, d)$ is locally compact.

\section{$3 \quad$ Heine-Borel metric spaces}

Let $(M, d)$ be a Heine-Borel metric space, i.e., a metric space such that a subset $K$ is compact if and only if it is bounded and closed. If we fix a point $x_{0} \in M$, then $M=\cup_{n \in \mathbb{N}} B\left[x_{0}, n\right]$. As $(M, d)$ is a Heine-Borel metric space, all closed metric balls are compact. Therefore $M$ is separable and $C(M)$ is a metric space with respect to the compact-open topology (cf. [8]) with the distance $\Delta$ defined by

$$
\Delta(f, g)=\sum_{n \in \mathbb{N}} \frac{1}{2^{n}} \frac{d_{n}(f, g)}{1+d_{n}(f, g)}
$$

where $d_{n}(f, g)=\sup _{x \in B\left[x_{0}, n\right]} d(f(x), g(x))$. For $f \in C(M)$, we denote

$$
\alpha(f)=\inf _{x \neq y} \frac{d(f(x), f(y))}{d(x, y)} \quad \text { and } \quad \beta(f)=\sup _{x \neq y} \frac{d(f(x), f(y))}{d(x, y)}
$$

When $f \in H(M, d)$ then $f$ is onto and $\alpha(f)=\beta(f)=\lambda(f)>0$. The following lemma is essential for the results in the next section: 
Lemma 3.1. For all $w, z \in M$ and all $a, b, c>0$, the set

$V[w, z, a, b, c]=\{f \in C(M): f$ is onto, $a \leq \alpha(f) \leq \beta(f) \leq b$ and $d(f(w), z) \leq c\}$

is compact with respect to the compact-open topology.

Proof. For all $f \in V=V[w, z, a, b, c]$ and all $x \in M$, we have $d(f(x), z) \leq$ $d(f(x), f(w))+d(f(w), z) \leq b d(x, w)+c$, and, since $M$ is Heine-Borel, $V(x)=$ $\{f(x): f \in V\}$ is relatively compact in $M$. Since for all $f \in V$, we have $d(f(x), f(y)) \leq b d(x, y)$, then $V$ is a uniformly equicontinuous family and so, by Arzelá-Ascoli's theorem for spaces that are an increasing countable union of compact subsets (cf. [8]), $V$ is relatively compact in $C(M)$ with respect to the compact-open topology. It remains to prove that $V$ is closed with respect to the compact-open topology. Let $\bar{f} \in \operatorname{cl}(V)$, where $\operatorname{cl}(V)$ is the closure of $V$ in $C(M)$, with respect to the compact-open topology, and let $\left(f_{n}\right)_{n \in \mathbb{N}}$ be a sequence in $V$ such that $f_{n} \rightarrow \bar{f}$. By taking limits in the inequalities of (4), we have $a \leq \alpha(\bar{f}) \leq \beta(\bar{f}) \leq b$ and $d(\bar{f}(w), z) \leq c$. To show that $f$ is onto, let $y \in M$. Since each $f_{n}$ is onto, there is a sequence $\left(x_{n}\right)_{n \in \mathbb{N}}$ in $M$ such that $f_{n}\left(x_{n}\right)=y$. Hence $d\left(x_{n}, y\right) \leq a^{-1} d\left(f_{n}\left(x_{n}\right), f_{n}(y)\right)=a^{-1} d\left(y, f_{n}(y)\right)$. Since $f_{n}(y) \rightarrow \bar{f}(y)$, we obtain that $\left(x_{n}\right)_{n \in \mathbb{N}}$ is a bounded sequence in the Heine-Borel space $M$. Hence there are $x \in M$ and a subsequence $\left(x_{n_{k}}\right)_{k \in \mathbb{N}}$ such that $x_{n_{k}} \rightarrow x$. Thus $d(\bar{f}(x), y) \leq d\left(\bar{f}(x), f_{n_{k}}(x)\right)+d\left(f_{n_{k}}(x), f_{n_{k}}\left(x_{n_{k}}\right)\right) \leq d\left(\bar{f}(x), f_{n_{k}}(x)\right)+b d\left(x, x_{n_{k}}\right)$, and, since $f_{n_{k}}(x) \rightarrow \bar{f}(x)$ and $x_{n_{k}} \rightarrow x$, it follows that $d(\bar{f}(x), y)=0$. Therefore $\bar{f}$ is onto showing that $V$ is compact with respect to the compact-open topology.

We have that $\log :(0, \infty) \rightarrow \mathbb{R}$ is a continuous homomorphism from the multiplicative group $(0, \infty)$ to the additive group $\mathbb{R}$. Thus the mapping $f \mapsto$ $\log (\lambda(f))$ is a continuous homomorphism from $H(M, d)$ to the additive group $\mathbb{R}$ and we can define a distance $\delta$ equivalent to $\Delta$ (cf. [8]) by

$$
\delta(f, g)=\Delta(f, g)+d\left(f\left(x_{0}\right), g\left(x_{0}\right)\right)+|\log (\lambda(f))-\log (\lambda(g))| .
$$


We denote the closed metric ball of $(H(M, d), \delta)$ centered in $f \in H(M, d)$ with radius $r>0$ by $\mathcal{B}[f, r]=\{g \in H(M, d): \delta(f, g) \leq r\}$.

The next result is an extension of the van Dantzig and van der Waerden's result for the group of homotheties $H(M, d)$ when $(M, d)$ is Heine-Borel:

Theorem 3.1. If $(M, d)$ is a Heine-Borel metric space, then $H(M, d)$ is a Heine-Borel metric space.

Proof. We have to show that $\mathcal{B}[\mathrm{id}, r]$ is compact for all $r>0$, where id is the identity mapping. This is done by showing that $\mathcal{B}[\mathrm{id}, r] \subset V[w, z, a, b, c]$, for some $V[w, z, a, b, c]$ like in Lemma 3.1. For all $f \in \mathcal{B}[\mathrm{id}, r]$, we have $d\left(f\left(x_{0}\right), x_{0}\right) \leq$ $r$ and

$$
|\log (\lambda(f))|=\mid \log (\lambda(f))-\log (\lambda(\text { id })) \mid \leq r
$$

Thus $\exp (-r) \leq \lambda(f) \leq \exp (r)$ and $\mathcal{B}[\mathrm{id}, r] \subset V=V\left[x_{0}, x_{0}, \exp (-r), \exp (r), r\right]$. It remains to prove that $\mathcal{B}[\mathrm{id}, r]$ is closed in $C(M)$, with respect to the compactopen topology, and hence compact. Let $\left(f_{n}\right)_{n \in \mathbb{N}} \subset \mathcal{B}[\mathrm{id}, r]$ be a sequence such that $f_{n} \rightarrow f$. The compactness of $V$ implies that $f$ is onto. By taking limits, $\delta(\mathrm{id}, f) \leq r$ and, for all $x, y \in M$, with $x \neq y$ we have

$$
\frac{d(f(x), f(y))}{d(x, y)}=\lim \frac{d\left(f_{n}(x), f_{n}(y)\right)}{d(x, y)}=\lim \lambda\left(f_{n}\right)=\lambda(f)
$$

and therefore $f \in \mathcal{B}[\mathrm{id}, r]$.

The following corollary is a immediately consequence of Proposition 2.1 and Theorem 2.1.

Corollary 3.1. Let $(M, d)$ be a Heine-Borel space. Then we have:

1. $I(M, d)$ is a Heine-Borel metric space.

2. $I(M, d)$ is a Lie group if, and only if, $H(M, d)$ is a Lie group.

The action of a group of transformations $G$ on a metric space $M$ is proper if, and only if, for all $w, z \in M$ there are neighborhoods $U_{w}$ and $U_{z}$ of $w$ and $z$, 
respectively, such that the subset $\left\{g \in G: g U_{w} \cap U_{z} \neq \emptyset\right\}$ is relatively compact with respect to the compact-open topology. The theorem below is a special case (for Heine-Borel spaces $(M, d)$ ) of a result on the action of $I(M, d)$ on $M$ that can be found in [13]:

Theorem 3.2. If $(M, d)$ is a Heine-Borel metric space, then the action of $I(M, d)$ on $M$ is proper.

Proof. Let $w, z \in M, U_{w}=B[w, 1], U_{z}=B[z, 1]$. Take $f \in\{g \in I(M, d)$ : $\left.g U_{w} \cap U_{z} \neq \emptyset\right\}$. Then there is $y \in U_{w}$ such that $f(y) \in U_{z}$. Therefore $d(f(w), z) \leq d(f(w), f(y))+d(f(y), z) \leq d(w, y)+d(f(y), z) \leq 2$. Hence $\left\{g \in I(M, d): g U_{w} \cap U_{z} \neq \emptyset\right\} \subset V[w, z, 1,1,2]$ and, by Lemma 3.1, is relatively compact with respect to the compact-open topology.

Remark 3.1. It is well known that Theorem 3.2 is not true if the group of isometries is replaced by the group of homotheties $H(M, d)$. For instance, let the space of real numbers $\mathbb{R}$ be endowed with the usual distance and take the sequence $\left(g_{n}\right)_{n \geq k}$ with $g_{n}(x)=n x$. We see that it does not have any convergent subsequence in the compact-open topology. On the other hand, since 0 is a fixed point for all $g_{n}$, there is $k \in \mathbb{N}$ such that

$$
\left(g_{n}\right)_{n \geq k} \subset\left\{g \in H(M, d): g U_{0} \cap U_{1} \neq \emptyset\right\},
$$

where $U_{0}$ and $U_{1}$ are arbitrary neighborhoods, respectively, of 0 and 1.

\section{Heine-Borel ultrametric spaces}

In this section, we consider $(M, d)$ be a Heine-Borel ultrametric metric space, i.e., a metric space where

$$
d(x, z) \leq \max \{d(x, y), d(y, z)\},
$$

for all $x, y, z \in M$. Given a subset $F \subset I(M, d)$ of isometries and $x \in M$, we let $\langle F\rangle$ be the subgroup of isometries generated by $F$ and $F(x)=\{f(x): f \in F\}$, the orbit of $x$ by $F$. 
Lemma 4.1. Take $x \in M$ and let $G=\langle F\rangle$, where $F \subset I(M, d)$. If $F(x) \subset$ $B[x, r]$, where $r>0$, then $G(x) \subset B[x, r]$ and hence $c l(G)$ is a compact subgroup.

Proof. If $f \in F$ then $d\left(f^{-1}(x), x\right)=d\left(f^{-1}(x), f^{-1}(f(x))\right)=d(x, f(x)) \leq r$. Let $g \in G$ be such that $g=f_{1} \cdots f_{n}$ with $f_{i} \in F$ or $f_{i}^{-1} \in F, i \in\{1, \cdots, n\}$. We proceed by induction on $n$ to show that $g(x) \in B[x, r]$. If $n=1$, by the previous inequality, $g(x) \in B[x, r]$. Assume that the result is true for $n$. Let $g=f_{1} \cdots f_{n} f_{n+1}$ and define $h=f_{1} \cdots f_{n}$. Hence

$$
\begin{aligned}
d(g(x), x)=d\left(h\left(f_{n+1}(x)\right), x\right) & \leq \max \left\{d\left(h\left(f_{n+1}(x)\right), h(x)\right), d(h(x), x)\right\}=(8) \\
& =\max \left\{d\left(f_{n+1}(x), x\right), d(h(x), x)\right\} \leq r,
\end{aligned}
$$

by the induction hypothesis, and for all $g \in G=\langle F\rangle, g(x) \in B[x, r]$. Therefore $G(x) \subset B[x, c]$ and $\operatorname{cl}(G)(x)$ is compact. The properness of the action implies that $\operatorname{cl}(G)$ is a compact subgroup (see [14] and also [13]).

The next corollary states that if $G \subset I(M, d)$ has the algebraic property to be finitely generated then the closure of $G$ has the topological property to be compact:

Corollary 4.1. Let $(M, d)$ a Heine-Borel ultrametric space. If $G \subset I(M, d)$ is finitely generated then $\operatorname{cl}(G)$ is a compact subgroup.

Proof. We have $G=\langle F\rangle$, where $F=\left\{f_{1}, \cdots, f_{n}\right\}$. Hence for all $i \in\{1, \cdots, n\}$ we have $d\left(f_{i}(x), x\right) \leq \max \left\{d\left(f_{1}(x), x\right), \cdots, d\left(f_{n}(x), x\right)\right\}=r$. By the Lemma 4.1, $\operatorname{cl}(G)$ is a compact subgroup.

Finally we have the following improvement of a theorem due to Gao and Kechris [13]:

Theorem 4.1. Let $(M, d)$ a Heine-Borel ultrametric space. $I(M, d)=\bigcup G_{n}$, where $G_{n}$ are compact subgroups such that $G_{n} \subset G_{n+1}$. 
Proof. Let $x \in M$ and, for each $n \in \mathbb{N}$, we define

$$
G_{n}=\{f \in I(M, d): d(f(x), x) \leq n\} .
$$

By Lemma 4.1 we have that $\left\langle G_{n}\right\rangle=G_{n}$ and $\operatorname{cl}\left(G_{n}\right)$ is compact. But it is easy to verify that $G_{n}$ is closed in $I(M, d)$ with respect to the compact-open topology, showing that $G_{n}$ is a compact subgroup. Clearly, we also have $G_{n} \subset G_{n+1}$ and $I(M, d)=\bigcup G_{n}$.

Theorem 4.1 also shows that, when $(M, d)$ is Heine-Borel ultrametric space, $I(M, d)$ is an amenable group (cf. [17]).

\section{$5 \quad \Sigma(M)$ quasi-compact}

Let $(M, d)$ be a locally compact metric space and $C(M)$ the set of all continuous maps of $M$ into itself. The following four lemmas are generalizations of some results of Manoussos-Strantzalos [9]. First we define

$$
V[a, b]=\{f \in C(M): f \text { is onto, } a \leq \alpha(f) \leq \beta(f) \leq b\} .
$$

where $a, b>0$ and $\alpha(f), \beta(f)$ are as in Equation (3). $V[a, b]$ is clearly an uniformly equicontinuous family of $C(M)$.

Lemma 5.1. For all $a, b>0$ and for all $V \subset V[a, b]$, let $V(x)=\{f(x): f \in$ $V\}$. Then the set

$$
K(V)=\{x \in M: V(x) \text { is relatively compact }\},
$$

is an open and closed subset of $M$.

Proof. The fact that $K(V)$ is open, is a consequence of the uniform equicontinuity of $V \subset C(M)$. In fact, take $x \in K(V)$ and for $y \in \operatorname{cl}(V(x))$ let $\varepsilon_{y}>0$ be such that $B\left(y, \varepsilon_{y}\right)$ is relatively compact. We see that $\left\{B\left(y, \varepsilon_{y}\right)\right\}_{y \in c l(V(x))}$ is a covering of the compact set $\operatorname{cl}(V(x))$. Hence there are $y_{i} \in \operatorname{cl}(V(x))$, 
$i \in\{1, \cdots, m\}$, such that $\left\{B\left(y_{i}, \varepsilon_{y_{i}}\right)\right\}_{i \in\{1, \cdots, m\}}$ is a finite subcovering. If we take $\delta<b^{-1} \inf d(u, v)$, where $u \in \operatorname{cl}(V(x))$ and $v \in M \backslash \bigcup_{i \in\{1, \cdots, m\}} B\left(y_{i}, \varepsilon_{y_{i}}\right)$, then $V(z) \subset \bigcup_{i \in\{1, \cdots, m\}} B\left(y_{i}, \varepsilon_{y_{i}}\right)$, for all $z \in B(x, \delta)$ and therefore $B(x, \delta) \subset K(V)$. We prove now that $K(V)$ is closed. For all $N \subset M$, we define $B(N, \varepsilon)=$ $\bigcup_{x \in N} B(x, \varepsilon)$. Take $x \in \operatorname{cl}(K(V))$ and let $\varepsilon>0$ be such that $B\left(x, \frac{5 b}{a} \varepsilon\right)$ is relatively compact, and $y \in K(V) \cap B(x, \varepsilon)$. Hence $\operatorname{cl}(V(y)) \subset B(V(B(x, \varepsilon)), a \varepsilon) \subset$ $V(B(x, 2 \varepsilon))$, because for each $y, z$ such that $d(y, x)<\varepsilon$ and $d(z, f(y))<a \varepsilon$, for some $f \in V$, then $d\left(f^{-1}(z), x\right) \leq d\left(f^{-1}(z), y\right)+d(y, x) \leq a^{-1} d(z, f(y))+$ $d(y, x)<2 \varepsilon$ and therefore $f^{-1}(z) \in B(x, 2 \varepsilon)$, so $z \in V(B(x, 2 \varepsilon))$. By the compactness of $\operatorname{cl}(V(y))$ we can get a finite subset $F \subset V$ such that $\operatorname{cl}(V(y)) \subset$ $F(B(x, 2 \varepsilon))$. We show that $V(x)$ is contained in the relatively compact set $F\left(B\left(x, \frac{5 b}{a} \varepsilon\right)\right)$. Let $f \in V$ and $g \in F$ such that $f(y) \in g(B(x, 2 \varepsilon)) \subset B(g(x), 2 b \varepsilon)$. Hence

$$
\begin{aligned}
d(f(x), g(y)) & \leq d(f(x), f(y))+d(f(y), g(x))+d(g(x), g(y)) \\
& \leq b d(x, y)+d(f(y), g(x))+b d(x, y) \leq 4 b \varepsilon
\end{aligned}
$$

and therefore $f(x) \in B(g(y), 4 b \varepsilon) \subset g\left(B\left(y, \frac{4 b}{a} \varepsilon\right)\right) \subset g\left(B\left(x, \frac{5 b}{a} \varepsilon\right)\right) \subset F\left(B\left(x, \frac{5 b}{a} \varepsilon\right)\right)$. Thus $x \in K(V)$ and the proof is concluded.

For the next lemmas, we assume that the space $\Sigma(M)$ of the connected components of $M$ is quasi-compact (compact but not necessarily Hausdorff) with respect to quotient topology. Under this assumption $M$ is separable and therefore is an increasing countable union of compact subsets, so that we can apply the Arzelá-Ascoli theorem (cf. [8]).

Lemma 5.2. There is a neighborhood $V$ of the identity in $H(M, d)$ that is relatively compact in $C(M)$ with respect to the compact-open topology.

Proof. For all $x \in M$, let $r_{x}>0$ be such that $B\left(x, 2 r_{x}\right)$ is relatively compact and take $y_{x} \in M$ with $x \neq y_{x}$, and $r_{x} \leq \frac{1}{4} d\left(x, y_{x}\right)$. Define

$$
V_{x}=\left\{f \in H(M, d): d(f(x), x) \leq r_{x} \text { and } d\left(f\left(y_{x}\right), y_{x}\right) \leq r_{x}\right\}
$$


Then $V_{x}$ is a neighborhood of the identity in $H(M, d)$ with respect to the compact-open topology. By the triangular inequality, for all $f \in V_{x}$, we have $d\left(f(x), f\left(y_{x}\right)\right) \leq 2 r_{x}+d\left(x, y_{x}\right) \leq \frac{3}{2} d\left(x, y_{x}\right)$ and $d\left(x, y_{x}\right) \leq 2 r_{x}+d\left(f(x), f\left(y_{x}\right)\right)$, which implies that $\frac{1}{2} d\left(x, y_{x}\right) \leq d\left(x, y_{x}\right)-2 r_{x} \leq d\left(f(x), f\left(y_{x}\right)\right)$. Hence $\frac{1}{2} \leq$ $\lambda(f) \leq \frac{3}{2}$. Therefore $V_{x} \subset V\left[\frac{1}{2}, \frac{3}{2}\right]$ and $x \in K\left(V_{x}\right)$. By Lemma 5.1, $K\left(V_{x}\right)$ is an open and closed subset of $M$ and therefore it is the union of connected components of $M$. Hence $q\left(K\left(V_{x}\right)\right)$ is an open subset of $\Sigma(M)$, where $q: M \rightarrow \Sigma(M)$ is the canonical projection, and $\left\{q\left(K\left(V_{x}\right)\right)\right\}_{x \in M}$ is an open covering of $\Sigma(M)$. Since $\Sigma(M)$ is quasi-compact, there are $x_{i} \in M, i \in\{1, \cdots, m\}$ such that $\left\{q\left(K\left(V_{x_{i}}\right)\right)\right\}_{i \in\{1, \cdots, m\}}$ is a finite open subcovering. We define $V=\bigcap_{i \in\{1, \cdots, m\}} V_{x_{i}}$ so that $V$ is also a neighborhood of the identity in $H(M, d)$ with respect to the compact-open topology. Therefore, $V(x) \subset V_{x_{i}}(x)$ is relatively compact for all $x \in M$. Since $V$ is clearly an equicontinuous family, by Arzelá-Ascoli theorem, $V$ is relatively compact in $C(M)$.

The preceding lemma shows that there is a neighborhood of the identity in $H(M, d)$ which is relatively compact in $C(M)$ with respect to the compact-open topology. For our purpose, namely to prove that $H(M, d)$ is locally compact, it sufficient to show that this neighborhood is also closed and therefore its closure is contained in $H(M, d)$. The following two lemmas are used to prove what is the most delicate part of the proof: the limit of a sequence in this neighborhood is onto:

Lemma 5.3. Let $V$ be the neighborhood of the identity as in Lemma 5.2. If a sequence $\left(f_{n}\right)_{n \in \mathbb{N}} \subset V$ is such that $f_{n} \rightarrow f$ with respect to the compact-open topology, then its image $f(M)$ is an open and closed subset of $M$.

Proof. By Lemma 5.1, it is sufficient to show that $f(M)=K(L)$, where $L=$ $\left\{f_{n}^{-1}, n \in \mathbb{N}\right\}$, because $L^{-1} \subset V \subset V\left[\frac{1}{2}, \frac{3}{2}\right]$. One one hand, since $d\left(x, f_{n}^{-1}(x)\right) \leq$ $2 d\left(f_{n}(x), f(x)\right)$ and $f_{n}(x) \rightarrow f(x)$, we have $f_{n}^{-1}(f(x)) \rightarrow x$ and $f(x) \in K(L)$ for all $x \in M$, because $M$ is locally compact. On the other hand, if $y \in K(L)$ 
then $F(y)$ is relatively compact in $M$, so there are $x \in M$ and a subsequence $\left(f_{n_{k}}^{-1}(y)\right)_{k \in \mathbb{N}}$ such that $f_{n_{k}}^{-1}(y) \rightarrow x$. Hence we have $d(f(x), y) \leq d\left(f(x), f_{n_{k}}(x)\right)+$ $d\left(f_{n_{k}}(x), y\right) \leq d\left(f(x), f_{n_{k}}(x)\right)+\frac{3}{2} d\left(x, f_{n_{k}}^{-1}(y)\right)$ and, since $f_{n_{k}}(x) \rightarrow f(x)$, we have $y=f(x)$.

If $M$ is connected then $f(M)=M$ and therefore $f$ is onto. But, as the following lemma shows, this is also true in the more general situation when $\Sigma(M)$ is quasi-compact with respect to the quotient topology:

Lemma 5.4. Let $V$ be the neighborhood of the identity as in Lemma 5.2. If a sequence $\left(f_{n}\right)_{n \in \mathbb{N}} \subset V$ is such that $f_{n} \rightarrow f$ with respect to the compact-open topology, then $f$ is onto.

Proof. Let $y \in M$. We denote by $S_{y}$ the connected component of $y$ and, for all $n \in \mathbb{N}$, we let $S_{n}$ be the component of $f_{n}^{-1}(y)$. Since each $f_{n}$ is a homeomorphism, we have $f_{n}\left(S_{n}\right)=S_{y}$ for all $n \in \mathbb{N}$. First we assume that $\left(S_{n}\right)_{n \in \mathbb{N}}$ has a constant subnet $\left(S_{n_{k}}=S_{0}\right)_{k \in \mathbb{N}}$, for some $S_{0} \in \Sigma(M)$. Hence $f_{n_{k}}\left(S_{0}\right)=S_{y}$, for all $k \in \mathbb{N}$. Let $x_{0} \in S_{0}$. Hence $f_{n_{k}}\left(x_{0}\right) \in S_{y}$ and, since $f_{n_{k}}\left(x_{0}\right) \rightarrow f\left(x_{0}\right)$, we have $f\left(x_{0}\right) \in S_{y}$. By Lemma 5.3, $S_{y} \subset f(M)$ and therefore $y \in f(M)$. Now we suppose that $\left(S_{n}\right)_{n \in \mathbb{N}}$ has no constant subnet. Hence, with the above notation, we can apply the following lemma that was proved in Manoussos-Strantzalos [9]:

Lemma 5.5. If $\left(S_{n}\right)_{n \in \mathbb{N}}$ has no constant subnet then there are $x_{0} \in M, a$ subsequence $\left(S_{n_{k}}\right)_{k \in \mathbb{N}}$ and a sequence $\left(x_{k}\right)_{k \in \mathbb{N}}$ such that $x_{k} \in S_{n_{k}}$ and $x_{k} \rightarrow x_{0}$.

By Lemma 5.5, there are $x_{0} \in M$, a subsequence $\left(S_{n_{k}}\right)_{k \in \mathbb{N}}$ and a subsequence $\left(x_{k}\right)_{k \in \mathbb{N}}$ such that $x_{k} \in S_{n_{k}}=f_{n_{k}}^{-1}\left(S_{y}\right)$ and $x_{k}=f_{n_{k}}^{-1}\left(y_{k}\right) \rightarrow x_{0}$, where $y_{k} \in S_{y}$. Hence

$$
\begin{aligned}
d\left(f\left(x_{0}\right), y_{k}\right) & \leq d\left(f\left(x_{0}\right), f_{n_{k}}\left(x_{0}\right)\right)+d\left(f_{n_{k}}\left(x_{0}\right), y_{k}\right) \\
& \leq d\left(f\left(x_{0}\right), f_{n_{k}}\left(x_{0}\right)\right)+\frac{3}{2} d\left(x_{0}, f_{n_{k}}^{-1}\left(y_{k}\right)\right)
\end{aligned}
$$


and, since $f_{n_{k}}\left(x_{0}\right) \rightarrow f\left(x_{0}\right)$, we have $y_{k} \rightarrow f\left(x_{0}\right)$ and therefore $f\left(x_{0}\right) \in S_{y}$. By Lemma 5.3, $S_{y} \subset f(M)$ and therefore $f$ is onto.

The following is a generalization of a result due to Manoussos and Strantzalos [9] for the group of homotheties:

Theorem 5.1. Let $(M, d)$ be a locally compact metric space such that the space of connected components $\Sigma(M)$ is quasi-compact with respect to the quotient topology. Then $H(M, d)$ is locally compact with respect to the compact-open topology.

Proof. Let $V=\bigcap_{i \in\{1, \cdots, m\}} V_{x_{i}} \subset C(M)$ be the relatively compact neighborhood of the identity as in Lemma 5.2. It is sufficient to show that $V$ is closed with respect to the compact-open topology. Let $\left(f_{n}\right)_{n \in \mathbb{N}} \subset V$ be a sequence such that $f_{n} \rightarrow f$. By Lemma 5.4, $f$ is onto. Arguing as in Lemma 3.1, we have that $d(f(x), f(y))=\lambda(f) d(x, y)$, where $\lambda(f)=\lim \lambda\left(f_{n}\right)$. Clearly, we also have $d\left(f\left(x_{i}\right), x_{i}\right) \leq r_{x_{i}}$ and $d\left(f\left(y_{x_{i}}\right), y_{x_{i}}\right) \leq r_{x_{i}}$, for all $i \in\{1, \cdots, m\}$ and therefore $f \in V$. Hence $V$ is compact with respect to the compact-open topology.

As a immediately corollary, we have a generalization of the classical theorem of van Dantzig and van der Waerden for the group of homotheties $H(M, d)$.

Corollary 5.1. Let $(M, d)$ be a connected and locally compact metric space. Then $H(M, d)$ is locally compact with respect to the compact-open topology.

The following corollary is a immediately consequence of Theorem 2.1.

Corollary 5.2. Let $(M, d)$ be a locally compact metric space such that the space of connected components $\Sigma(M)$ is quasi-compact with respect to the quotient topology. Then $I(M, d)$ is a Lie group if, and only if, $H(M, d)$ is a Lie group. 


\section{Quasi-metric spaces}

Let $d: M \times M \rightarrow[0, \infty)$ satisfying the distance axioms except perhaps that $d(x, y)$ is not necessary equal to $d(y, x)$ for all $x, y \in M$. If the topologies generated by the forward metric balls $B_{+}(x, r)=\{y \in M: d(x, y)<r\}$ and generated by the backward metric balls $B_{-}(x, r)=\{y \in M: d(y, x)<r\}$ are the same, we call $(M, d)$ a quasi-metric space and $d$ a quasi-distance. The group of homotheties $H(M, d)$ and the group of isometries $I(M, d)$ are defined in the same way as for metric spaces. An interesting example of such spaces are the Finsler manifolds where its Finsler function $F$ is positively homogeneous but not necessary absolutely homogeneous. The notation of the forward and backward metric balls follows the book of D. Bao, S.S. Chern and Z. Shen [1]. The following corollary is an extension of Theorem 5.1 for quasi-metric spaces:

Corollary 6.1. Let $(M, d)$ be a locally compact quasi-metric space and suppose that its space of connected components $\Sigma(M)$ is quasi-compact with respect to the quotient topology. Then $H(M, d)$ is locally compact with respect to the compactopen topology.

Proof. If $(M, d)$ is a quasi-metric space then the function $\bar{d}: M \times M \rightarrow[0, \infty)$, defined by $\bar{d}(x, y)=d(x, y)+d(y, x)$, is a distance and it is straightforward to verify that the topology of $(M, \bar{d})$ is the same as the topology of $(M, d)$. So, $(M, \bar{d})$ is a locally compact metric space and its space of connected components $\Sigma(M)$ is quasi-compact with respect to the quotient topology. It also straightforward to verify that $H(M, d)$ is a closed subgroup of $H(M, \bar{d})$ with respect to the compact-open topology and hence the proof is concluded.

\section{Applications}

The following theorems are immediately consequences of Corollary 5.2 and its version for quasi-metric spaces. 


\subsection{Finsler manifolds}

Let $(M, d)$ be a connected Finsler manifold (cf. [1]), where its Finsler function $F$ is positively homogeneous but not necessary absolutely homogeneous, i.e., $F(x, a v)=a F(x, v)$, for all $v \in T_{x}(M)$, only if $a \geq 0$. We define, for all $x, y \in M, d(x, y)$ as the greatest lower bound of the length of all smooth curves joining $x$ to $y$. As we noted in the previous section, $(M, d)$ is a quasi-metric space but $d$ is not necessary a distance [1]. Recently, Shaoqiang Deng and Zixin Hou (cf. [5]) proved the following generalization of a classical result of Riemannian geometry due to Myers and Steenrod [11]: if $(M, d)$ is a connected Finsler manifold then its group of isometries is a Lie group with respect to the compact-open topology. Thus we have the following result:

Theorem 7.1. If $(M, d)$ is a connected Finsler manifold, then $H(M, d)$ is a Lie group.

\subsection{Singular spaces}

Let $(M, d)$ be a length space, that is, a metric space where the distance between every two points in it is realized as the infimum of lengths of curves joining them. Alexandroff generalized the concept of sectional curvature of a Riemannian manifold to such spaces using the conclusion of Topogonov's comparison theorem as the definition (cf. [2]). A length space which is locally compact, of finite Hausdorff dimension and whose curvature is nowhere $-\infty$ is called an Alexandroff space. It was proved by Fukaya-Yamaguchi [6] that $I(M, d)$ is a Lie group if $(M, d)$ is an Alexandroff space. Thus we have the following result:

Theorem 7.2. If $(M, d)$ is an Alexandroff space, then $H(M, d)$ is a Lie group.

Finally, let $(M, d)$ be a Hadamard space, that is, a complete simply connected length space with nonpositive curvature. For such space $(M, d)$ the ideal boundary $M(\infty)$ is defined as the set of equivalence classes of rays in $(M, d)$ with the natural topology. The space $M(\infty)$ has a natural metric $T d$ called Tits metric, which measures the deviation of $(M, d)$ from flatness (cf. [2]). 
It was proved by Yamaguchi [16] that if $(M, d)$ is locally compact, geodesically complete Hadamard space and $(M(\infty), T d)$ is compact, then $I(M, d)$ and $I(M(\infty), T d)$ are Lie groups. Thus we have the following result:

Theorem 7.3. If $(M, d)$ is a locally compact, geodesically complete Hadamard space and $(M(\infty), T d)$ is compact, then $H(M, d)$ is a Lie group.

We remark that the compactness of $(M(\infty), T d)$ is equivalent to the hypothesis that the Tits topology coincides with the natural topology.

\section{References}

[1] Bao, D. Chern, S. S. and Shen, Z., An Introduction to Riemann-Finsler Geometry. Graduate Text Math. 200, Springer, Berlin-New York (2000).

[2] Bridson, M. R. and Haefliger, A., Metric Spaces of Non-Positive Curvature, Fund. Princ. Math. Sc. 319, Springer, Berlin (1999).

[3] Cassels, J. W. S., Local Fields. L.M.S. Student Text 3, Cambridge Un. Press, Cambridge, (1986).

[4] van Dantzig, D. e van Waerden, B. L., Über metrische homogene Räume. Abh. Math. Sem. Hamburg 6 (1928), 367-376.

[5] Deng, S. and Hou, Z., The Group of Isometries of a Finsler Space. Pacific Journal of Mathematics 207 (2002), 149-155.

[6] Fukaya, K. and Yamaguchi, T., Isometry groups of singular spaces. Math. Z. 216 (1994), 31-44.

[7] Kobayashi, S. and Nomizu, K., Foundations of Differential Geometry. Interscience Publishers. New York (1963).

[8] Lima, E. L., Espaços Métricos. Projeto Euclides. IMPA. Rio de Janeiro (1977). 
[9] Manoussos, A. and Strantzalos, P., On the Group of Isometries on a Locally Compact Metric Space. Journal of Lie Theory 13 (2003), 7-12.

[10] Montgomery, D. and Zippin, L., Topological Transformation Groups. Interscience. London (1955).

[11] Myers, S. B. and Steenrod, N., The group of isometries of a Riemannian manifold. Ann. of Math. 40 (1939), 400-416.

[12] Schafarevich I. R. and Borevich, Z. I., Number Theory, Academic Press. New York (1966).

[13] Su Gao and Kechris, A. S., On the Classification of Polish Metric Spaces Up to Isometry. Mem. of Am. Math. Soc. 161 (2003).

[14] Strantzalos, P., Actions by Isometries. Lecture Notes in Math. 1375 (1989), 319-325.

[15] Yamabe, H., A generalization of a theorem of Gleason. Ann. Math. 58 (1953), 351-365.

[16] Yamaguchi, T., Isometry groups of spaces with curvature bounded above. Math. Z. 232 (1999), 275-286.

[17] Zimmer, R. J., Ergodic Theory and Semisimple Groups. Monographs in Math 81, Birkhäuser, Basel (1984).

Instituto de Matemática

Universidade Estadual de Campinas

Cx. Postal 6065

13.081-970 Campinas-SP, Brasil 